Copyright (C 2014 IEEE. Personal use of this material is permitted. Permission from IEEE must be obtained for all other uses, in any current or future media, including reprinting/republishing this material for advertising or promotional purposes, creating new collective works, for resale or redistribution to servers or lists, or reuse of any copyrighted component of this work in other works. 


\title{
Sensitivity of Electric Vehicles Demand Profile to the Batteries Departure State-of-Charge
}

\author{
Ehsan Pashajavid, Farhad Shahnia \\ Department of Electrical and Computer Engineering, Curtin University \\ Perth, Australia
}

\begin{abstract}
This paper focuses on the impacts of considering batteries state-of-charge (SOC) at the departure time on the demand modeling of plug-in electric vehicles (PEVs). Almost all of the previous researches assumed that PEVs batteries at the departure time are fully charged; however, this assumption is highly questionable because it is probable for a PEV to not be charged every day. The probability density function of a vehicle owners' willingness to fulfill the daily charging is extracted according to the initial SOC of a PEV and the estimated distance of its next trip. Afterwards, with the aim of considering the uncertainties with the associated random variables as well as properly adjusting vehicles SOC at the departure time, a Monte Carlo based multi loop (MCML) algorithm is developed which is composed of two loops, namely the inner loop and the outer loop. In order to implement the proposed stochastic method, a case study has been conducted employing the gathered datasets related to the ICE vehicles in Tehran. Appropriate Student's $t$ copula functions have been fitted to the datasets in order to take into account the correlation structure among them as well as to generate the required random samples.
\end{abstract}

Index Terms--Demand modeling, Distribution system, Electric vehicles, Monte Carlo simulation, Smart grid, Uncertainty.

\section{INTRODUCTION}

In the recent decade, electrification of the transportation sector was actively pursued throughout the world mainly due to environmental concerns and energy security challenges [1-2]. Estimating the distribution network demand profile is of great importance for distribution network operators in network planning. Different criteria need to be considered when planning the network, among which integration of Plug-in Electric Vehicles (PEVs) is a very new criterion [3-6]. Several researches have already proved that electric network assets, especially the distribution transformers may experience overloading problems in the presence of high clusters of PEVs in an area [7-9]. Some improvement methods have been proposed [10-11]. In addition, the voltage unbalance issues due to the unequal distribution of PEVs in different modes of operation are studied [12].

Different methodologies are utilized in modeling the electric load demand of PEVs in order to achieve more reliable and realistic estimations. However, there are some challenges about load demand derivation of networks in the presence of PEVs which were not considered in literature. As an example, in the studies of [13-15], the state-of-charge (SOC) of PEV batteries at the arrival time was calculated based on the daily travelled distance of a PEV. However, the main assumption behind these analyses is the fully-charged status of PEVs at their departure time in that day. This assumption for level I (slow) charging in residential locations is highly questionable as it is not always valid but has a significant effect on the analysis results

SOC at the departure time depends on several parameters such as SOC at the arrival time, battery capacity, the rating of a battery charger, available charging time and occurrence of a daily charging. Among these parameters, SOC at arrival and occurrence of a daily charging associate SOC at departure to the charging history of a PEV, which are investigated in detail in this paper. Considering the probability of a daily charging occurrence significantly influences the SOC at departure. This parameter is also ignored in the literature of previous researches [16-19]. In practice, it is the vehicle owner who decides whether to charge his car, based on the current SOC and the expected distance of his next trip. If he decides not to charge the car, the SOC at departure of the PEV in the following day is identical to the SOC at arrival. Ignoring such probabilities when modeling and estimating PEVs load demand may result in non-accurate results which in turn, lead to problematic consequences in system planning such as over/under estimation costs or overloading of distribution network assets.

Regarding various uncertainties in PEVs, demand modeling of this vehicular load is carried out via probabilistic and stochastic methodologies [20-22]. The random variables considered in demand estimation of PEVs are their arrival time, travelled distance and departure time. Correlation of these random variables (RVs) in probabilistic methodologies has significant impacts on the analysis output and thus, should be evaluated precisely. Therefore, multivariate Probability Density Functions (PDF) should be utilized in order to consider this correlation. In case the associated RVs do not follow identical distributions, copula functions are applied to fit the appropriate joint distributions to the datasets [22-25].

In this paper, a multivariate probabilistic method is proposed in order to extract the load profiles in the distribution network in the presence of PEVs, considering the impact of $\mathrm{SOC}$ at the departure on the modeling procedure. First, in order to model uncertainties with the datasets, the joint probability distributions are extracted by utilizing a Student's t copula. Afterwards, the PDF associated with the occurrence of the daily charging is fitted considering the SOC at arrival as well as the distance of the next trip. The demand modeling procedure is accomplished through a multi-loop Monte Carlo 
analysis by using the synthetic data generated through the extracted joint PDFs.

The remainder of this paper is organized as follows. Section II explains the employed multivariate modeling methodology. Section III presents the Monte Carlo based algorithm, used for calculating the estimated PEV load profiles. The sensitivity of the extracted demand profiles to SOC at departure are illustrated in section IV.

\section{UNCERTAINTY MODELING}

\section{A. Theory: Student's t copula}

Probabilistic modeling of multivariate systems is utilized to integrate the correlation among the associated random variables. However, if similar distribution type is not followed by the individual variables, extraction of the joint distributions cannot be carried out via conventional methods. Copula functions are deployed in such scenarios. Copulas refer to the class of multivariate distribution functions supported on the unit cube with uniform marginals. In fact, copulas link the marginal densities of RVs to a joint density with a complex dependency structure specified in a single function. Student's $t$ copula is a well-known form of elliptical copulas and is highly efficient in examining realistic datasets because of its heavy tails [25]. In other words, the Student's $t$ copula presents more observations in the tails than the Gaussian one.

For every random variable $\mathbf{x}=\left(x_{1}, \ldots, x_{p}\right) \in \mathfrak{R}^{p}$, copula functions $C_{\mathbf{R}}:\left[\begin{array}{ll}0 & 1\end{array}\right]^{p}-\left[\begin{array}{ll}0 & 1\end{array}\right]$ are utilized to correlate univariate continuous marginal Cumulative Distribution Functions (CDFs) to a joint CDF as

$$
C_{\mathbf{R}}\left(F_{1}\left(x_{1}\right), F_{2}\left(x_{2}\right), \ldots, F_{p}\left(x_{p}\right)\right)=H\left(x_{1}, x_{2}, \ldots, x_{p}\right)
$$

where $F_{l}\left(x_{1}\right), \ldots, F_{p}\left(x_{p}\right)$ and $H\left(x_{1}, \ldots, x_{p}\right)$ are respectively the marginal and joint CDFs. The standardized joint CDF of Student's $t, H_{t, \boldsymbol{\rho}, v}$, is represented as

$$
\begin{gathered}
h_{t, \mathbf{\rho}, v}(\mathbf{x})=\frac{\Gamma\left(\frac{v+p}{2}\right) \mid \mathbf{\rho}^{-\frac{1}{2}}(v \pi)^{\frac{p}{2}}}{\Gamma\left(\frac{v}{2}\right)\left(1+\frac{1}{v}(\mathbf{x}-\boldsymbol{\mu})^{T} \mathbf{\rho}^{-1}(\mathbf{x}-\boldsymbol{\mu})\right)^{\frac{v+p}{2}}} \\
H_{t, \mathbf{\rho}, v}\left(x_{1}, x_{2}, \ldots, x_{p}\right)=\int_{-\infty}^{x_{1}} \int_{-\infty}^{x_{2}} \ldots \int_{-\infty}^{x_{p}} h_{t, \mathbf{\rho}, v} d x_{1} d x_{2} \ldots d x_{p}
\end{gathered}
$$

where $h_{t, \boldsymbol{\rho}, v}$ is the multivariate Student's $t$ PDF, $\Gamma$ represents the Gamma function, $v$ is degree of freedom and $\boldsymbol{\mu}$ is vector of means. Moreover, the linear correlation $\boldsymbol{\rho}$ is a symmetric, positive definite matrix with unity diagonal members. Hence, for any $\mathbf{u}=\left(u_{1}, u_{2}, \ldots, u_{p}\right) \in\left[\begin{array}{ll}0 & 1\end{array}\right]^{p}$ the Student's $t$ copula, $C_{\boldsymbol{\rho}, v}^{t}$, is defined as

$$
\begin{aligned}
C_{\mathbf{p}, v}^{t}\left(u_{1}, u_{2}, \ldots, u_{p}\right) & =H_{t, \mathbf{p}, v}\left(F_{t, v}^{-1}\left(u_{1}\right), F_{t, v}^{-1}\left(u_{2}\right), \ldots, F_{t, v}^{-1}\left(u_{p}\right)\right) \\
& =\int_{-\infty}^{F_{t, v}^{-1}\left(u_{l}\right)} \int_{-\infty}^{F_{t, v}^{-1}\left(u_{2}\right)} \ldots \int_{-\infty}^{F_{t, v}^{-1}\left(u_{p}\right)} h_{t, \mathbf{p}, v} d x_{1} d x_{2} \ldots d x_{p}
\end{aligned}
$$

where $F_{t, v}^{-1}$ is the inverse Student's $t$ CDF.

\section{B. System datasets}

This section concerns with extraction of a joint distribution that is used to generate the required system synthetic data. The datasets include departure time (DEP), travelled distance (TRAV) and arrival time (ARR) of the randomly selected ICE vehicles, employed for commuting in Tehran. Figure 1 shows these datasets along with the best fitted PDFs. As seen from this figure, a Weibull distribution as (5) is fitted to DEP while TRAV and ARR are modeled using Generalized Extreme Value PDFs as (6). Table I shows the parameters of these PDFs which are suggested as the most appropriate functions to be fitted to the associated random variables. The obtained PDFs are utilized as the marginal distributions to derive a multivariate PDF.

$f_{\mathrm{Wbl}}(x)=\frac{\beta}{\alpha}\left(\frac{x}{\alpha}\right)^{(\beta-1)} e^{-\left(\frac{x}{\alpha}\right)^{\beta}} \quad, x>0$

$f_{\mathrm{Gev}}(x)=\frac{1}{\gamma}\left(1+\zeta \frac{(x-\lambda)}{\gamma}\right)^{-\left(1+\frac{1}{\zeta}\right)} e^{-\left(1+\zeta \frac{(x-\lambda)}{\gamma}\right)^{-\frac{1}{\zeta}}}$

The correlation among these random variables is as follows

$\boldsymbol{\rho}=\left[\begin{array}{ccc}1 & -0.48 & -0.31 \\ -0.48 & 1 & 0.39 \\ -0.31 & 0.39 & 1\end{array}\right]$

Employing (4), a Student's $t$ copula is utilized to define the joint CDF of the random variables, $H$ (DEP, TRAV, ARR), through relating their univariate marginal distributions, $F_{\text {DEP }}(\mathrm{DEP}), F_{\text {TRAV }}(\mathrm{TRAV})$ and $F_{\text {ARR }}(\mathrm{ARR})$ as

$C^{t}\left(F_{\text {DEP }}, F_{\text {TRAV }}, F_{\text {ARR }}, \boldsymbol{\rho}_{3 \times 3}\right)=H(\mathrm{DEP}, \mathrm{TRAV}, \mathrm{ARR})$

\section{THE Proposed METHOdOLOGY}

\section{Modeling of Charging Occurrence per Day}

Although it can be assumed that PEVs are charged every day by the owners, in reality, this depends on the SOC at the arrival time $\left(\mathrm{SOC}_{\text {init }}\right)$ as well as the estimated travel distance of the next trip. In other words, a number of PEVs would not be plugged into the grid after arriving at home. Consequently, their SOC at the next departure $\left(\mathrm{SOC}_{\mathrm{dep}}\right)$ will be equal to their $\mathrm{SOC}_{\text {init }}$. Considering the probability of daily charging occurrence in load demand modeling of PEVs has significant effect on the results.
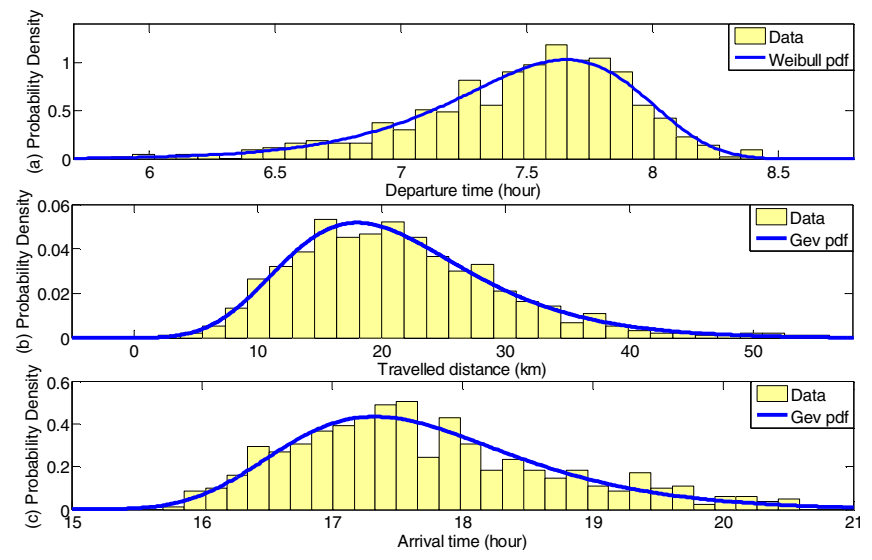

Figure 1. The data and PDFs of a) home departure time DEP, b) daily travelled distance TRAV and c) home arrival time ARR. 
TABLE I. PARAMETERS OF THE FITTED PDFS

\begin{tabular}{|ll|}
\hline \hline Datasets & The suggested PDF \\
\hline DEP $_{\mathrm{T}}$ & $\alpha=7.67454, \beta=21.3812$ \\
\hline TRAV $_{\mathrm{D}}$ & $\xi=-0.052368, \lambda=17.6568, \gamma=7.1222$ \\
\hline ARR $_{\mathrm{T}}$ & $\xi=-0.060798, \lambda=17.2700, \gamma=0.84832$ \\
\hline \hline
\end{tabular}

A bivariate PDF is derived in this section to determine whether a PEV will be charged on a specific day. The fitted PDF is later utilized to explain PEV owners' willingness to perform a daily charging. Due to lack of a general historical data on PEVs, the state-of-fuel of conventional ICE vehicles at the refueling time $\left(\mathbf{S O F}_{\text {init }}\right)$ as well as the anticipated distance of their upcoming trips $\left(\mathbf{T R A} \mathbf{V}^{e}\right)$ are utilized here to define $\mathbf{S O C}_{\text {init }} \cdot \mathbf{S O F}$ init $_{\text {is }}$ transformed to its equivalent $\mathbf{S O C}$ init, denoted by $\mathbf{S O C}_{\text {init }}^{e}$, as

$\mathrm{SOC}_{\text {init }}^{e}=\frac{\mathrm{SOF}_{\text {init }} \times \mathrm{TANK}_{\mathrm{CAP}} \times \mathrm{EFF}_{\mathrm{ICE}}}{\mathrm{EFF}_{\mathrm{DRV}} \times \mathrm{BATT}_{\mathrm{CAP}}}$

where $\mathrm{TANK}_{\mathrm{CAP}}$ and $\mathrm{EFF}_{\mathrm{ICE}}$ are the fuel tank capacity (in Liter) and the driving efficiency coefficient (in $\mathrm{km} / \mathrm{Liter}$ ) of the considered ICE vehicles, respectively. Similarly, BATT ${ }_{\mathrm{CAP}}$ and $\mathrm{EFF}_{\mathrm{DRV}}$ are the battery capacity (in $\mathrm{kWh}$ ) and the driving efficiency coefficient (in $\mathrm{km} / \mathrm{kWh}$ ) of PEVs, respectively. Then, a bivariate distribution is employed to model the derived $\mathbf{S O C}_{\mathrm{init}}^{\mathrm{e}}$ as well as the related TRAV $\mathbf{V}^{e}$ of the ICE vehicles. Eventually, a PDF is constructed that is used to determine whether a PEV will be charged on a specific day according to its $\mathrm{SOC}_{\text {init }}$ and TRAV.

Figure 2 illustrates $\mathbf{T R A V}^{e}$ along with the related SOC $_{\text {init }}^{\mathrm{e}}$ extracted from (8). Empirical PDFs $f_{\text {SOC }_{\text {init }}}\left(\right.$ SOC $\left.^{e}\right)$ and $f_{\text {TRAV }}$ (TRAV $\left.^{e}\right)$ are fitted to these variables based on their characteristics. Since these distributions are not in the same type and the correlation between them is not ignorable, a bivariate Student's $t$ copula is utilized to correlate the univariate marginal distributions, $F_{\text {SOC }_{\text {init }}}\left(\mathrm{SOC}^{e}\right)$ and $F_{\text {TRAV }^{\mathrm{e}}}\left(\mathrm{TRAV}^{e}\right)$, to the joint distribution function of $H\left(\mathrm{SOC}^{\mathrm{e}}, \mathrm{TRAV}^{\mathrm{e}}\right)$ as

$$
C^{t}\left(F_{\text {SOC }_{\text {init }}^{e}}, F_{\text {TRAV }^{e}}, \boldsymbol{\rho}_{2 \times 2}\right)=H\left(\mathrm{SOC}^{e}, \mathrm{TRAV}^{e}\right)
$$

Accordingly, the bivariate PDF shown in Fig. 3 is derived by employing the generated random samples which is used in determining the probability of PEV charging occurrence with respect to $\mathrm{SOC}_{\text {init }}$ and TRAV.
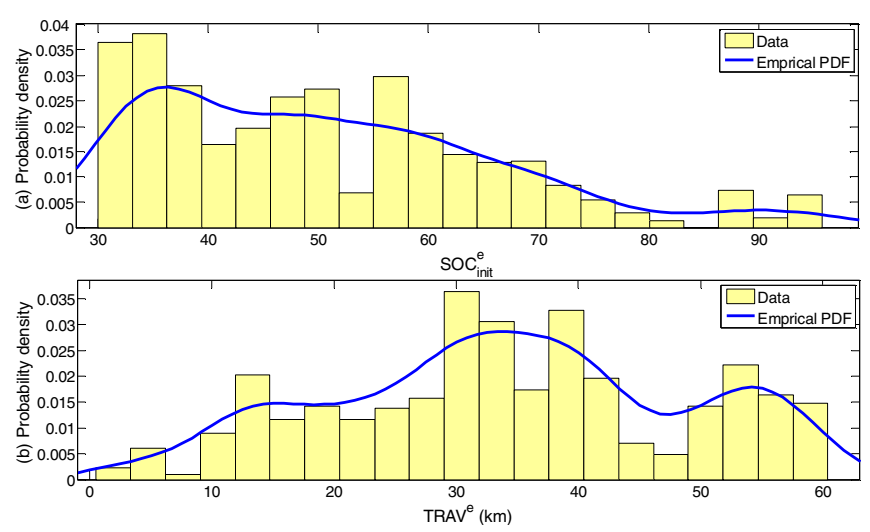

Figure 2. The data and empirical PDFs of a) SOC $\mathbf{C i n i t}_{\text {it }}^{\mathrm{e}}$ and b) TRAV $\mathbf{T}^{e}$.

\section{MCML algorithm}

A Monte Carlo-based multi-loop (MCML) algorithm is utilized in this paper for modeling. This algorithm has two loops, namely the inner loop and the outer loop. The inner loop is designed to derive the hourly power consumption of PEVs for a specific $S_{\text {dep }}$. Moreover, the occurrence probability of a daily charging as well as the uncertainty modeling of the related RVs is considered. The inner loop is assumed to have 2000 iterations which is suitable to satisfy the latter target.

The outer loop extracts the demand profiles through modifying $\mathrm{SOC}_{\text {dep }}$ for the inner loop. After each inner loop, the expected SOCs are calculated which are utilized as $\mathrm{SOC}_{\text {dep }}$ for the next iteration in the outer loop.

The flowchart of the MCML algorithm is shown in Fig. 4 and the relevant steps of this algorithm are described briefly below:

Step 1) Sample Generation: Random samples are generated from (7) in order to consider uncertainties with the RVs. Figure 5 shows scatter plots of the synthetic data along with the actual datasets. It is obvious that the generated random samples have the same marginal and correlation characteristics of the actual datasets. As expected from the correlation matrix $\boldsymbol{\rho}$, there is a tendency for small (large) values of DEP to be associated with large (small) values of both TRAV and ARR. Further, there is a tendency for small (large) values of TRAV to be associated with small (large) values of ARR.

Step 2) Calculation of $\mathrm{SOC}_{\text {init }}: \mathrm{SOC}_{\text {init }}$ is calculated for each $\mathrm{PEV}$ based on $\mathrm{SOC}_{\mathrm{dep}}$ from

$\mathrm{SOC}_{\text {init }}=\mathrm{SOC}_{\text {dep }}-\frac{\mathrm{TRAV}_{n}}{\mathrm{EFF}_{\mathrm{DRV}} \times \mathrm{BATT}_{\mathrm{CAP}}} \times 100$

Note that, at the first iteration of the outer loop, $\mathrm{SOC}_{\text {dep }}$ is assumed as $100 \%$. For the next iterations, this parameter is modified according to the SOCs in the previous iterations.

Step 3) Determination of Charging Occurrence per day: Daily charging occurrence is calculated for PEVs. The occurrence probability is derived according to $\mathrm{SOC}_{\text {init }}$ and $\mathrm{TRAV}^{e}$ of a PEV from Fig. 3.

Step 4) Calculation of hourly power demand: Different technical parameters of the PEVs such as batteries Depth-ofDischarge (DOD), charging power of a battery $\left(\mathrm{P}_{\mathrm{Chg}}\right)$ and efficiency coefficient of a battery charger $\left(\mathrm{EFF}_{\mathrm{Chg}}\right)$ are considered in this step for calculation of the hourly power demand of PEVs in the network. These parameters can be

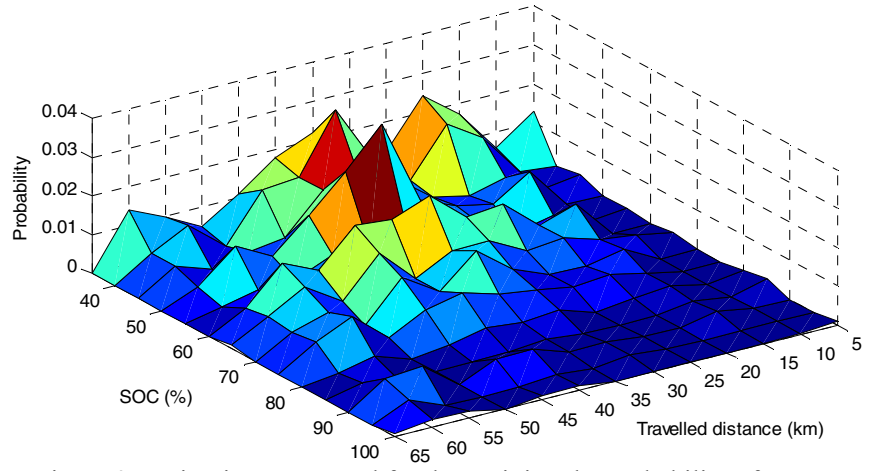

Figure 3. Bivariate PDF used for determining the probability of a PEV charging per a specific day according to its $\mathrm{SOC}_{\text {init }}$ and TRAV $\mathrm{TH}^{e}$. 


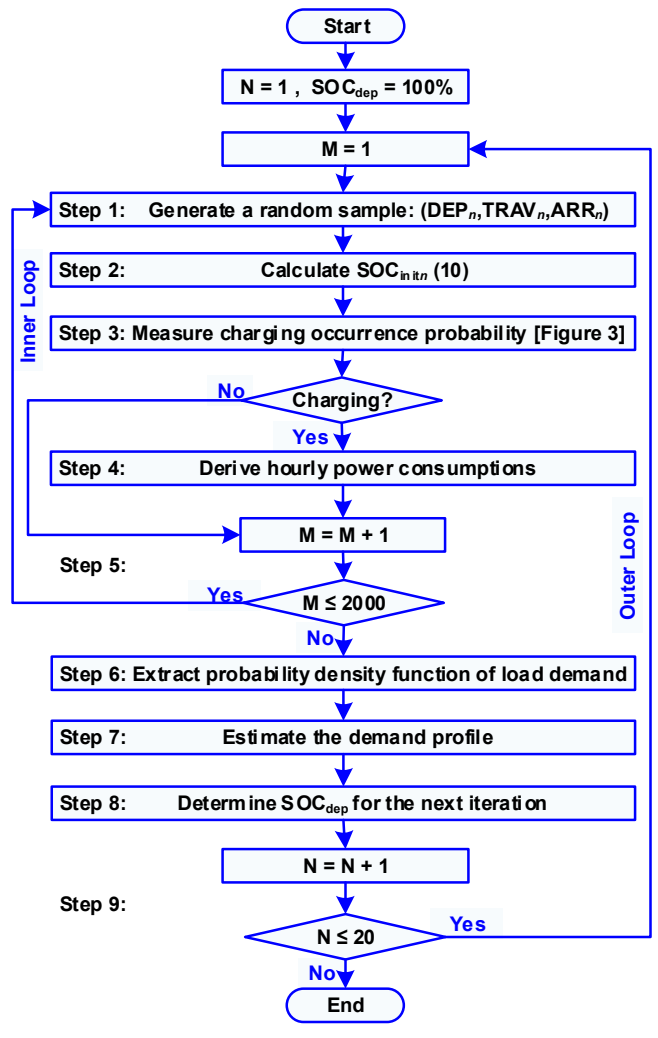

Figure 4. The developed Monte Carlo-based multi-loop algorithm for modeling and analysis of the expected load demand from PEVs

TABLE II. CONSIDERED PEV PARAMETERS IN THE MODELLING

\begin{tabular}{|ll|}
\hline \hline DOD & $70 \%$ \\
\hline BATT $_{\text {CAP }}$ & $20 \mathrm{kWh}$ \\
\hline $\mathrm{P}_{\text {Chg }}$ & $3 \mathrm{kWh}$ \\
\hline $\mathrm{EFF}_{\text {Chg }}$ & $90 \%$ \\
\hline $\mathrm{EFF}_{\text {DRV }}$ & $3 \mathrm{~km} / \mathrm{kWh}$ \\
\hline \hline
\end{tabular}

found in Table II. Hourly power consumption of the PEVs is derived according to the following algorithm. This step is carried out in regard to the occurrence probability of the charging procedure, calculated in Step 3. In the following algorithm, $\mathrm{h}$ and $\triangle \mathrm{SOC}$ are hour and SOC increment respectively. In addition, $\mathrm{BATT}_{\text {Curve }}$ indicates a battery charging curve as a function of its SOC.

$$
\begin{aligned}
& \text { Start: } \mathrm{h}=\mathrm{ARR} \\
& \mathrm{SOC}^{\mathrm{h}}=\mathrm{SOC}_{\text {init }} \\
& \text { Loop: }: \mathrm{P}_{\mathrm{Chg}}^{\mathrm{h}}=\mathrm{BATT}_{\text {Curve }}\left(\mathrm{SOC}^{\mathrm{h}}\right) / \mathrm{EFF}_{\mathrm{Chg}} \\
& \Delta \mathrm{SOC}=\left(\mathrm{BATT}_{\text {Curve }}\left(\mathrm{SOC}^{\mathrm{h}}\right) / \mathrm{BATT}_{\mathrm{CAP}}\right) \times 100 \\
& \mathrm{SOC}^{\mathrm{h}+1}=\mathrm{SOC}^{\mathrm{h}}+\Delta \mathrm{SOC} \\
& \text { if } \quad \mathrm{SOC}^{\mathrm{h}+1}<100 \rightarrow \text { Loop } \\
& \text { End }
\end{aligned}
$$

Figure 6 shows charging curve of a Lead-acid battery which is taken into account in this paper [10].

Step 5) Completion of the inner loop: Steps 1-4 are repeated until all the pre-specified number of samples are generated and examined. Mentioned earlier, in this study, 2000 randomly a)

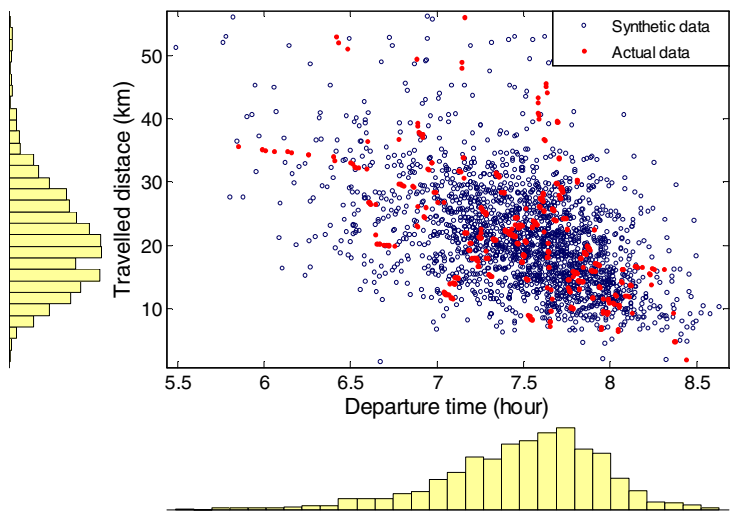

b)
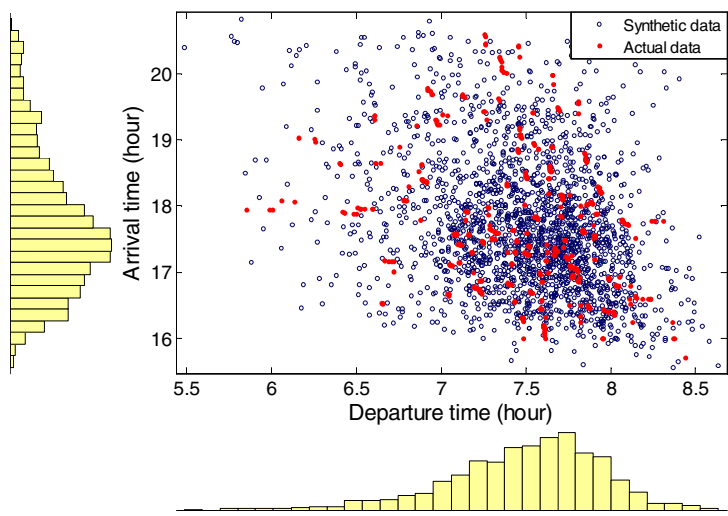

c)

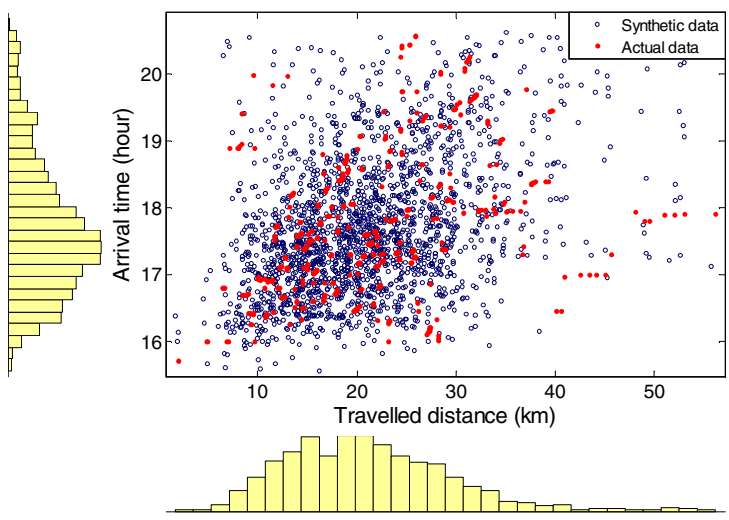

Figure 5. Scatter plots between a) departure time and travelled distance, b) departure and arrival time and c) travelled distance and arrival time.

generated samples are considered within each iteration to evaluate load demands extracted according to the assigned $\mathrm{SOC}_{\text {dep. }}$.

Step 6) Calculation of hourly distribution of consumed power: The hourly distribution of the consumed power is calculated from cumulative outcomes of Step 5. Then, the PDF of the charging load within each hour is derived through fitting the most appropriate distribution to the extracted power curves.

Step 7) Calculation of demand profile: The demand profile is derived by calculating the expected values of the extracted power curves in Step 6. Figure 7 shows box plots as well as the estimated demand profiles of some sample iterations of the outer loop. For instance, Fig. 7(c) is related to the derived demand profile of a PEV at the end of the second iteration of the outer loop when the occurrence probability of a daily charging is considered to be $75 \%$. 


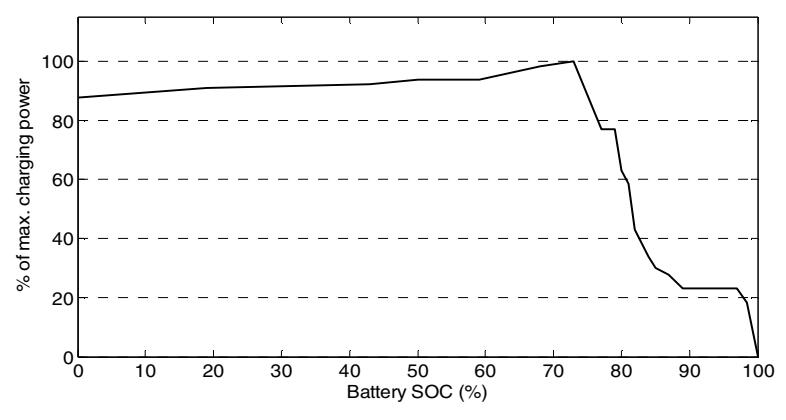

Figure 6. Charging curve of a Lead-acid battery
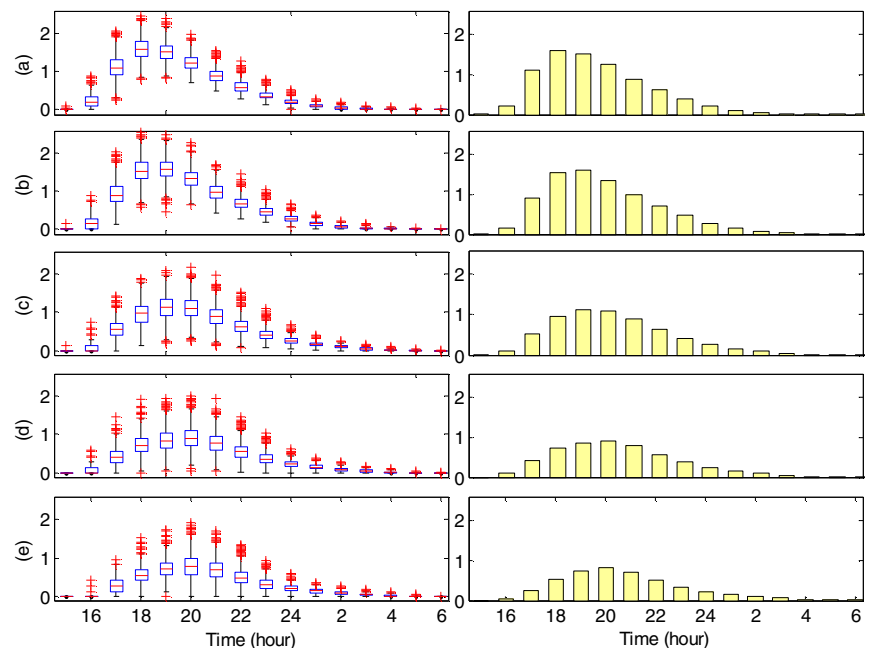

Figure 7. Sample box plots and demand profiles of a PEV (kW) with charging probability of a) $100 \%$, b) $85 \%$, c) $75 \%$, d) $65 \%$ and e) $50 \%$ at the $1^{\text {st }}, 4^{\text {th }}, 2^{\text {nd }}, 6^{\text {th }}$ and $2^{\text {nd }}$ iteration of the outer loop respectively.

TABLE III. EXTRACTED SOC DEP $_{\text {CONSIDERING THE RELATED }}$ CHARGING OCCURRENCE PROBABILITIES

\begin{tabular}{|cc|cc|}
\hline \hline $\begin{array}{c}\text { Occurrence } \\
\text { Probability (\%) }\end{array}$ & $\begin{array}{c}\text { SOC }_{\text {dep }} \\
\mathbf{( \% )}\end{array}$ & $\begin{array}{c}\text { Occurrence } \\
\text { Probability (\%) }\end{array}$ & SOC $_{\text {dep }} \mathbf{( \% )}$ \\
\hline 95 & 98 & 60 & $65 \& 88$ \\
\hline 90 & 94 & 55 & $60 \& 75$ \\
\hline 85 & $89 \& 96$ & 50 & $64 \& 69$ \\
\hline 80 & 91 & 45 & $58 \& 61$ \\
\hline 75 & 75 & 40 & 57 \\
\hline 70 & $70 \& 83$ & 35 & 53 \\
\hline \hline
\end{tabular}

Step 8) Determination of $\mathrm{SOC}_{\mathrm{dep}}$ : The expected value of the final SOCs is calculated. This value is used as the $\mathrm{SOC}_{\mathrm{dep}}$ in the next iteration of the outer loop.

Step 9) Completion of the outer loop: Steps 1-8 are repeated until the final demand profile is achieved. The proper amount of $\mathrm{SOC}_{\mathrm{dep}}$ to be used in estimating the demand profile of a specific system is obtained when the last iteration of the outer loop is carried out.

\section{ANALYSIS RESULTS}

Let us consider the PEVs in the system under study have the technical parameters given in Table I. The MCML algorithm, described in Section III is developed and utilized in this paper for system analysis. In this section, the outcomes are compared with each other regarding various charging occurrence probabilities in order to illustrate $\mathrm{SOC}_{\mathrm{dep}}$ impacts on the demand curves.

Figure 8 shows the extracted demand profiles within iterations of the outer loop besides to their related $\mathrm{SOC}_{\text {dep }}$. According to Fig. 8(a), when all the PEVs are charged every day, the estimated demand profile at the first iteration becomes identical to the outcome of the other iterations. However, in order to achieve more accurate demand profiles, it is essential to properly modify the $\mathbf{S O C}_{\text {dep }}$ through incorporating the outer loop of the proposed MCML algorithm. Figure 8 illustrates that the estimated load profiles with assuming fully charged batteries at the departure time are far different from the scenarios that different $\mathrm{SOC}_{\text {dep }}$ are considered. By applying the proposed algorithm, the proper $\mathrm{SOC}_{\text {dep }}$ under various charging occurrence probabilities are extracted and given in Table III. As an example, from this table, it is seen that in order to achieve a reliable demand profile with a charging occurrence probability of $90 \%$, the proper $\mathrm{SOC}_{\text {dep }}$ is $94 \%$. As shown in Fig. 8(c), it is possible to attain more than one demand profile under some occurrence probabilities. In such cases, all of the associated demand profiles should be taken into account.

\section{CONCLUSION}

A Monte Carlo-based multi-loop stochastic algorithm was developed and utilized in this paper to calculate the expected demand profiles of PEVs in electric networks. For this, first appropriate Student's $t$ copula functions were applied to generate the required synthetic data considering the correlation structure among the associated random variables. Then, the algorithm used to calculates the hourly power consumption of PEVs within its inner loop through utilizing the randomly generated samples. Later, the expected value of the SOCs was calculated to define the battery SOC at the departure time for the next iteration of the outer loop; which in turn, leads to adjusting the estimated demand profile. The developed method considered various occurrence probabilities of the daily charging for PEVs and addressed the sensitivity of the PEVs load demand to the battery SOC at the departure time. In this regard, the dependency of vehicle owners' behavior in performing daily charging to their SOC at the arrival time and the expected length of their next trips was modeled using a bivariate PDF.

Based on the simulation results, it was illustrated that the battery SOC at the departure time depends highly on PEV owners' willingness to fulfill the daily charging. The results from the developed algorithm verified that for more reliable load demand profiles, the SOC at departure time should be calculated based on the variables discussed in the paper and considering a fully-charged battery at the departure time will lead to far different estimates (even about 100\%) for the load demand profile in the network. 


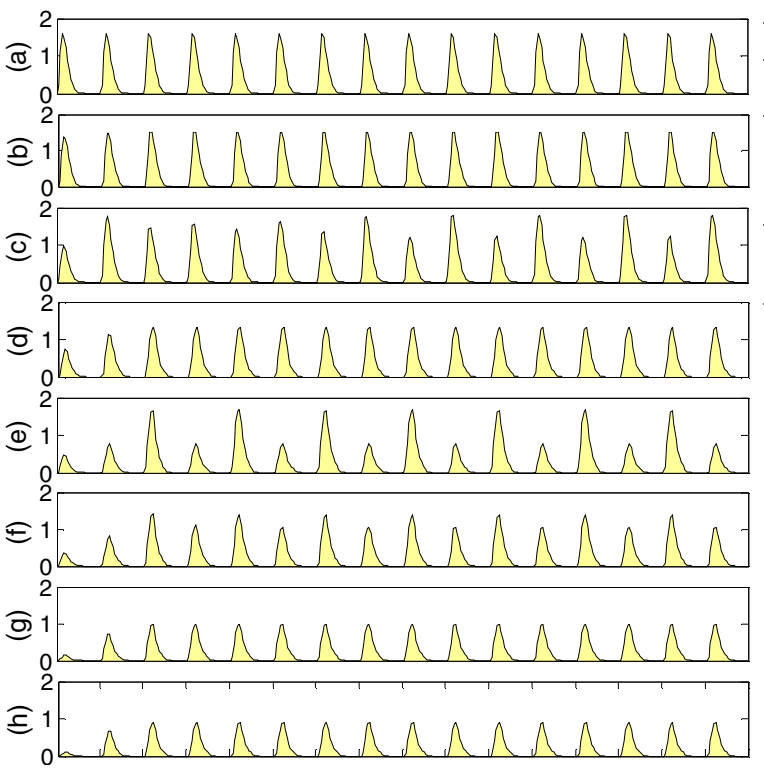

\section{REFERENCES}

[1] A.W. Su, H. Rahimi Eichi, W. Zeng, M. Chow, "A Survey on the Electrification of Transportation in a Smart Grid Environment," IEEE Trans. Ind. Informat., vol. 8, pp. 1-10, 2012.

[2] A.S. Masoum, S. Deilami, P.S. Moses, M.A.S. Masoum, A. Abu-Siada, "Smart load management of plug-in electric vehicles in distribution and residential networks with charging tations for peak shaving and loss minimisation considering voltage regulation," IET Gener. Transm. Distrib., vol. 5, pp. 877-888, 2011

[3] S. Shahidinejad, S. Filizadeh, E. Bibeau, "Profile of charging load on the grid due to plug-in vehicles," IEEE Trans. Smart Grid, vol. 3, pp. 135-141, 2012.

[4] G. Li, X.P. Zhang, "Modeling of plug-in hybrid elecrtic vehicle charging demand in probabilistic power flow calculations," IEEE Trans. Smart Grid, vol. 3, pp. 492-499, 2012.

[5] S. Shao, M. Pipattanasomporn, S. Rahman, "Demand response as a load shaping tool in an intelligent grid with electric vehicles," IEEE Trans. Smart Grid, vol. 2, pp. 624-631, 2011.

[6] A. Ashtari, E. Bibeau, S. Shahidinejad, T. Molinski, "PEV charging profile prediction and analysis based on vehicle usage data," IEEE Trans. Smart Grid, vol. 3, pp. 341-350, 2012.

[7] F. Shahnia, M.T., Wishart, A., Ghosh, G. Ledwich, F. Zare, "Smart demand side management of low-voltage distribution networks using multi-objective decision making," IET Gener. Transm. Distrib., vol. 6, pp. 986-1000, 2012.

[8] A.D. Hilshey, P.D.H. Hines, P. Rezaei, J.R. Dowds, "Estimating the Impact of Electric Vehicle Smart Charging on Distribution Transformer Aging," IEEE Trans. Smart Grid, vol. 4, pp. 905 - 913, 2013.

[9] J.M. Sexauer, K.D. McBee, K.A. Bloch, "Applications of Probability Model to Analyze the Effects of Electric Vehicle Chargers on Distribution Transformers," IEEE Trans. Power Systems, vol. 28, pp. 847-854, 2013.

[10] F. Shahnia, M.T. Wishart and A. Ghosh "Voltage regulation, power balancing and battery storage discharge control by smart demand side management and multi-objective decision making," Australasian Universities Power Engineering Conference (AUPEC), pp. 1-6, 2013.

[11] M. Moghbel, M.A.S. Masoum and F. Shahnia, "Coordinated Charging of PEVs in Unbalanced Residential Network Based on Worst Node Voltage Profile," IEEE Power \& Energy Society General Meeting (PES), pp. 1-6, 2013.

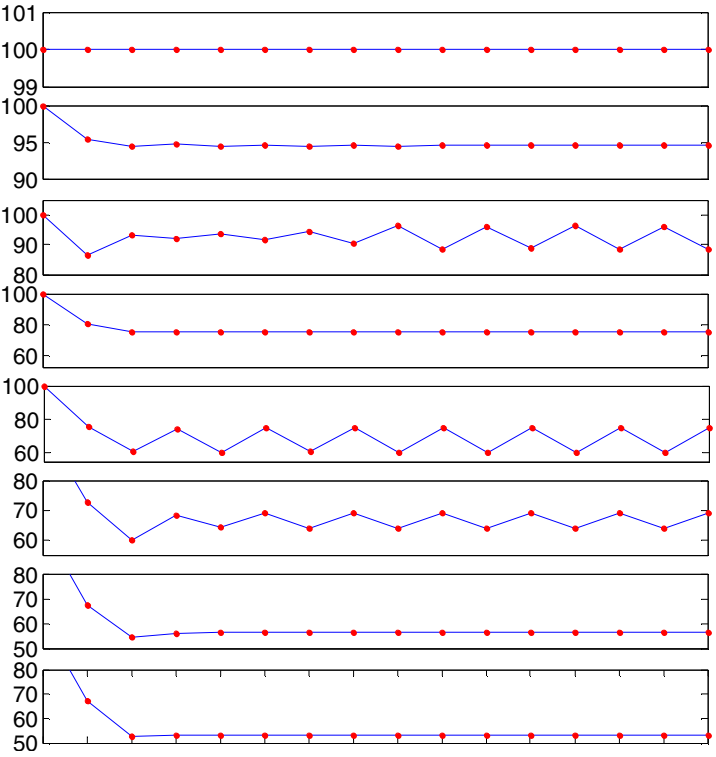

[12] F. Shahnia, A. Ghosh, G. Ledwich, and F. zare, "Predicting Voltage Unbalance Impacts of Plug-in Electric Vehicles Penetration in Residential Low-voltage Distribution Networks," Electric Power Components and Systems, Vol. 41, No. 16, 1594-1616, 2013.

[13] K. Qian, C. Zhou, M. Allan, Y. Yuan, "Modeling of load demand due to EV battery charging in distribution systems," IEEE Trans. Power Systems, vol. 26, pp. 802-810, 2010.

[14] Z. Darabi, M. Ferdowsi, "Aggregated impact of plug-in hybrid electric vehicles on electricity demand profile," IEEE Trans. Sustainable Energy, vol. 2, pp. 501-508, 2011.

[15] E.L. Karfopoulos, N.D. Hatziargyriou, "A Multi-Agent System for Controlled Charging of a Large Population of Electric Vehicles," IEEE Trans. Power Systems, vol. 28, pp. 1196-1204, 2013.

[16] K. Clement-Nyns, E. Haesen, J. Driesen, "The impact of charging plugin hybrid electric vehicles on a residential distribution grid," IEEE Trans. Power Systems, vol. 25, pp. 371-380, 2010.

[17] P. Richardson, D. Flynn, A. Keane, "Local Versus Centralized Charging Strategies for Electric Vehicles in Low Voltage Distribution Systems," IEEE Trans. Smart Grid, vol. 3, pp. 1020-1028, 2012.

[18] Z. Luo, Z. Hu, Y. Song, Z. Xu, H. Lu, "Optimal Coordination of Plugin Electric Vehicles in Power Grids With Cost-Benefit Analysis-Part II: A Case Study in China," IEEE Trans. Power Systems, vol. 28, pp. 3556-3565, 2013.

[19] Z. Liu, F. Wen, G. Ledwich, "Optimal Planning of Electric-Vehicle Charging Stations in Distribution Systems," IEEE Trans. Power Delivery, vol. 28, pp. 102-110, 2013.

[20] E. Pashajavid, M.A. Golkar, "Charging of plug-in electric vehicles: Stochastic modelling of load demand within domestic grids," $20^{\text {th }}$ Iranian Conf. on Electrical Engineering (ICEE2012), pp. 535-539.

[21] Rong-Ceng Leou, Chun-Lien Su, Chan-Nan Lu, "Stochastic Analyses of Electric Vehicle Charging Impacts on Distribution Network," IEEE Trans. Power Systems, vol. 29, pp. 1055-1063, 2014.

[22] E. Pashajavid, M.A. Golkar, "Charging of plug-in electric vehicles: Stochastic modelling of load demand within domestic grids," Electrical Power and Energy Systems, vol. 61, pp. 197-207, 2014.

[23] H. Valizadeh Haghi, M.A. Golkar, M. Tavakoli Bina, S.M. MoghaddasTafreshi, "Using Copulas for analysis of large datasets in renewable distributed generation: PV and wind power integration in Iran," Renewable Energies, vol. 35, pp. 1991-2000, 2010.

[24] A. Lojowska, D. Kurowicka, G. Papaefthymiou, L. van der Sluis, "Stochastic modeling of power demand due to EVs using Copula," IEEE Trans. Power Systems, vol. 27, pp. 1960-1968, 2012.

[25] R.B. Nelsen, An Introduction to Copulas, New York: Springer, 2006. 